\title{
Principio activo citotoxico de Espeletia killipii Cuatr. sobre células tumorales y su toxicidad frente a células normales humanas
}

\author{
Gustavo Jaimes $^{1,2}$, Clemencia de Castro ${ }^{1}$, Fabio Ancizar Aristizaba ${ }^{2}$, Tulia Riveros de Murcia ${ }^{1}$, \\ Rubén Torrenegra ${ }^{3}$, Alba N. Téllez Alfonso ${ }^{3 *}$ \\ ${ }^{1}$ Instituto Nacional de Cancerología E.S.E., Grupo de Investigación Nuevos Fármacos en Oncología, Bogotá, \\ Colombia, \\ ${ }^{2}$ Departamento de Farmacia, Universidad Nacional, Bogotá, Colombia, \\ ${ }^{3}$ Departamento de Química, Universidad Javeriana, Grupo de Investigación Fitoquímica, Bogotá, Colombia
}

\begin{abstract}
RESUMO: "Princípio ativo citotóxico de Espeletia killipii Cuatr. Sobre células tumorais e sua toxicidade frente a células normais humanas". De Espeletia killipi Cuart. foi isolado uma sesquiterpenlactona identificada como acetato de longipilina que mostrou atividade citotóxica. A citotoxidade foi avaliada em células normais obtidas de sangue periférico, tiróide, testículo e epitélio da boca. Células da medula óssea de pacientes com leucemia crônica mielóide, linfoma de Hodking (do Instituto Nacional de Câncer de Bogotá, Colômbia) e células K562 também foram avaliadas. A citotoxidade foi determinada através do teste MTT (3-(4,5-dimethyldiazol-2-yl)-2,5 diphenyl Tetrazolium Bromid). Os ensaios mostraram que a substância não é tóxica para células normais mas a $3 \mu \mathrm{g} / \mathrm{mL}$ apresentou significante atividade em células tumorais e linhagem K562. Conseqüentemente, lavando-se em conta essa significante ação, novas investigações podem ser consideradas plausíveis
\end{abstract}

Unitermos: Espeletia killipii, sesquiterpenolactona, atividade citotóxica.

\begin{abstract}
The compound responsible for the citotoxic effect was identified as longipilin acetate, a sesquiterpenelactone isolated from Espeletia killipi Cuatr. Citotoxicity was assessed by using normal cells obtained from peripheral blood, thyroid, testicle and mouth epithelium. Bone marrow cells from patients with chronic myeloid leukemia, Hodking lymphoma (from Cancer National Institute, Bogotá Colombia) and the cell line K562 were also assayed. Citotoxicity was determined by the MTT cell viability test (3-(4,5-dimethyldiazol-2-yl)-2,5 diphenyl Tetrazolium Bromid]. The assays revealed that the substance is risk-free on normal cells but at $3 \mu \mathrm{g} / \mathrm{mL}$ has significant activity on tumor cells and K562 cell line. Consequently, taken into account this significant action, new research approaches can be foreseen.
\end{abstract}

Keywords: Espeletia killipii, sesquiterpenelactone, cytotoxic activity.

\section{INTRODUCCIÓN}

El descubrimiento de nuevos agentes con actividad anticáncer se ha convertido en una de las metas de la investigación científica moderna; la molécula ideal para este fin debe eliminar ó incapacitar una variedad importante de subpoblaciones celulares de un tumor sin afectar en lo posible las del tejido normal (Huang; Oliff, 2001).

En trabajo anterior fue mostrado los efectos citotoxicos in vitro de extractos y fracciones de Espeletia killipii frente a lineas celulares tumorales humanos (Alfonso et al., 2006). En este trabajo, una sesquiterpenlactona aislada de la misma planta, presentó un potencial citotóxico importante sobre líneas celulares tumorales con valores de Concentración Citotóxica media $\left(\mathrm{CC}_{50}\right)$ menor de $1 \mu \mathrm{g} / \mathrm{mL}$ para las líneas celulares colombianas CSC-1170, CSC-1595, CSC-3322 y CSC$3325 ; \mathrm{CC}_{50}=1 \mu \mathrm{g} / \mathrm{mL}$ para MDA MB 435 y NCI- H23; para $\mathrm{MCF}-7$ una $\mathrm{CC}_{50}=2 \mu \mathrm{g} / \mathrm{mL}$ y una $\mathrm{CC}_{50}$ superior a $16 \mu \mathrm{g} / \mathrm{mL}$ para PC -3 y U-251. Es indispensable observar la toxicidad del principio activo frente a células normales y células obtenidas directamente de pacientes con cáncer; dentro del estudio de la molécula como posible agente antitumoral.

\section{MATERIAL Y METODOS}

El principio activo fue caracterizado e identificado por sus constantes físicas y por métodos espectroscópicos. El punto de fusión se determinó en un fusiómetro Melt Temp. Laboratory Devices; las rotaciones específicas en un polarímetro Polartronic Schmidt; el espectro infrarrojo tomados en un espectrofotómetro infrarrojo Shimadzu 710B; los espectros de masas en un espectrómetro de masas por impacto electrónico, EIMS 902, de doble enfoque, con inyección directa a $70 \mathrm{eV}$. Los espectros RMN ${ }^{1} \mathrm{H}, \mathrm{RMN}{ }^{13} \mathrm{C}$ y RMN 2D, 
tanto homonucleares como heteronucleares, $\mathrm{COSY}{ }^{1} \mathrm{H}-$ ${ }^{1} \mathrm{H}$ (Correlation Spectroscopy), NOESY ${ }^{1} \mathrm{H}-{ }^{1} \mathrm{H}$ (Nuclear Overhauser Effect Spectroscopy), HMBC (Heteronuclear Multiple Quantum Coherence), HMQC (Heteronuclear Multiple Quantum Coherence) y H-C COBI ) fueron tomados en un espectrómetro Bruker AMX-400 (400 $\mathrm{MHz}$ ). Los espectros fueron corridos en solución en $\mathrm{CDCl}_{3}$.

Se evaluó la toxicidad sobre cultivos celulares primarios de: células mononucleares de siete donantes, células sanas de: tiroides, testículo y boca, células mononucleares de médula ósea de dos pacientes con leucemia mieloide crónica y dos de linfoma de Hodking y la línea celular tumoral K562. Todas las muestras y líneas fueron suministradas por el Instituto Nacional de Cancerología E.S.E. de Bogotá, Colombia.

\section{Cultivos}

Se establecieron cultivos de: células mononucleares de sangre periférica y cultivos de testículo, tiroides y boca. Se realizaron curvas de crecimiento, en todos los casos, con el fin de calibrar el número adecuado de células a inocular en las cajas de 96 pozos para una absorbancia apropiada.

\section{Principio activo}

El compuesto fue suministrado por el Grupo de investigación Fitoquímica de la Pontificia Universidad Javeriana de Bogotá Colombia. Se prepararon soluciones seriadas de: $50 \mu \mathrm{g} / \mathrm{mL}, 25 \mu \mathrm{g} / \mathrm{mL}, 12.5 \mu \mathrm{g} / \mathrm{mL}, 6.0 \mu \mathrm{g} / \mathrm{mL}$ y $3.0 \mu \mathrm{g} / \mathrm{mL}$; utilizando como solvente dimetil sulfóxido (DMSO) al $0.2 \%$.

\section{Bioensayos}

Se utilizó el ensayo colorimétrico de proliferación celular de MTT (Sigma). Los cultivos se efectuaron en cajas de 96 pozos, con medio L-15 (SIGMA) suplementado con: $10 \%$ de suero fetal bovino (GIBCO) y $10 \%$ de penicilina-estreptomicina. Como control positivo se utilizó Doxorrubicina $\mathrm{HCl}$ a $0.07 \mu \mathrm{g} /$ $\mathrm{mL}$ (Pharmacía \& Uphjon).

Los datos obtenidos se evaluaron estadísticamente utilizando análisis de varianza y la prueba de Tuckey; las $\mathrm{CC}_{50}$ se estimaron utilizando el logaritmo de la concentración con el paquete estadístico SAS con plataforma para windows.

\section{RESULTADOS Y DISCUSIÓN}

El espectro IR del compuesto activo, presentó absorciones en $1780 \mathrm{~cm}^{-1}$, (lactona), en 1740 y $1240 \mathrm{~cm}^{-1}$ (grupo acetato) y en $1720 \mathrm{~cm}^{-1}$ (grupo $-\mathrm{C}=\mathrm{O}$, de éster). El espectro de $\mathrm{RMN}{ }^{1} \mathrm{H}$ confirma la presencia de la lactona mediante las señales en $\delta 6.3(\mathrm{~d}, \mathrm{~J}=3.42 \mathrm{~Hz}), 5.9(\mathrm{~d}$, $\mathrm{J}=2.44 \mathrm{~Hz}), 2.7(\mathrm{~m})$. El grupo metil éster se evidencia por la señal en $\delta 3.8$ y el grupo angelato por las señales en $\delta 1.7(\mathrm{~m}), 1.9$ y $1.7(\mathrm{~m})$ (Tabla 1). El espectro de RMN ${ }^{13} \mathrm{C}$, DEPT/COM mostró señales correspondientes a 8 grupos $\mathrm{CH}, 3$ grupos $\mathrm{CH}_{2}, 4$ grupos $\mathrm{CH}_{3}, 8$ carbonos

Tabla 1. Datos de $\mathrm{RMN}{ }^{1} \mathrm{H}$, tomados de los espectros del compuesto acetato de longipilina $(400 \mathrm{MHz})$ en $\mathrm{CDCl}_{3}$.

\begin{tabular}{cc}
\hline Hidrogeno & $\left(\delta_{\mathrm{H}, \mathrm{ppm})}\right.$ \\
\hline 1 & $7.1(\mathrm{dd}, \mathrm{J}=9.9 ; 9.0 \mathrm{~Hz})$ \\
$2^{\mathrm{a}}$ & $3.05(\mathrm{~m})$ \\
$2^{\mathrm{b}}$ & $2.4(\mathrm{~m})$ \\
$3^{\mathrm{a}}$ & $1.25(\mathrm{~m})$ \\
$3^{\mathrm{b}}$ & $2.6(\mathrm{~s})$ \\
5 & $2.7(\mathrm{~m})$ \\
6 & $4.25(\mathrm{dd}, \mathrm{J}=9.5 ; 9.7 \mathrm{~Hz})$ \\
7 & $2.9(\mathrm{dq}, \mathrm{J}=1.9 \mathrm{~Hz})$ \\
8 & $6.8(\mathrm{dd}, \mathrm{J}=0.98 ; 1.2 ; 9.7)$ \\
9 & $5.83(\mathrm{~d}, \mathrm{~J}=8.5 \mathrm{~Hz})$ \\
$13^{\mathrm{a}}$ & $5.9(\mathrm{~d}, \mathrm{~J}=2.4 \mathrm{~Hz})$ \\
$13^{\mathrm{b}}$ & $6.3(\mathrm{~d}, \mathrm{~J}=3.42)$ \\
15 & 1.7 \\
$\mathrm{MeO}$ & $3.8(\mathrm{~s})$ \\
$\mathrm{AcO}$ & $2.0(\mathrm{~s})$ \\
$\mathrm{Ang} \mathrm{O}$ & \\
$3^{\prime}$ & $6.1(\mathrm{~d})$ \\
$2^{\prime}$ & $1.5(\mathrm{~d})$ \\
$3^{\prime}$ & $1.9(\mathrm{~m})$ \\
\hline
\end{tabular}

a,b,c Señales intercambiables 
Tabla 2. Datos de $\mathrm{RMN}{ }^{13} \mathrm{C}$, tomados de los espectros del compuesto acetato de longipilina.

\begin{tabular}{ccc}
\hline $\mathrm{C}$ & $\mathrm{Tipo} \mathrm{de}$ & $\delta_{\mathrm{C}}$ \\
\hline 1 & $-\mathrm{CH}=$ & 149.2 \\
2 & $\mathrm{CH}_{2}$ & 24.4 \\
3 & $\mathrm{CH}_{2}$ & 35.1 \\
4 & $\mathrm{C}$ & 59.2 \\
5 & $\mathrm{CH}-\mathrm{O}$ & 62.5 \\
6 & $\mathrm{CH}-\mathrm{O}$ & 76.0 \\
7 & $\mathrm{CH}$ & 45.3 \\
8 & $\mathrm{CH}-\mathrm{O}$ & 70.4 \\
9 & $\mathrm{CH}-\mathrm{O} \mathrm{Ac}$ & 69.4 \\
10 & $\mathrm{C}-\mathrm{C}=\mathrm{O}$ & 126.4 \\
11 & $-\mathrm{C}=\mathrm{CH} 2$ & 133.4 \\
12 & $\mathrm{O}-\mathrm{C}=\mathrm{O}$ & 165.3 \\
13 & $=\mathrm{CH} 2$ & 122.5 \\
14 & $-\mathrm{COO}-$ & 165.7 \\
15 & $\mathrm{CH}$ & 17.2 \\
$\mathrm{MeO}$ & $\mathrm{CH}-\mathrm{O}-\mathrm{CO}$ & 52.1 \\
$\mathrm{AcO}$ & $\mathrm{C}=\mathrm{O}$ & 170.0 \\
& $\mathrm{CH}-\mathrm{COO}$ & 20.10 \\
1 & $-\mathrm{COO}-$ & 168.0 \\
2 & $-\mathrm{C}=\mathrm{C}$ & 129.0 \\
3 & $=\mathrm{CH}-$ & 139.7 \\
$\mathrm{Me}$ & $\mathrm{CH}-\mathrm{C}-$ & 20.4 \\
$\mathrm{Me}$ & $\mathrm{CH}-\mathrm{CH}$ & 15.4 \\
\hline
\end{tabular}

Tabla 3. Datos de RMN bidimensional H-C directo y HMBC, tomados de los espectros del compuesto acetato de longipilina.

\begin{tabular}{llll}
\hline $\mathrm{C}$ & $\delta_{\mathrm{C}}$ & $\delta_{\mathrm{H}} \mathrm{H}-\mathrm{C}$ directo & $\mathrm{HMBC}$ \\
\hline $\mathrm{CH}_{3}$ & 15.9 & $1.92 \mathrm{dq}$ & $126.7,140.3,166.1$ (débil) \\
$\mathrm{CH}_{3}$ & 17.7 & $1.68 \mathrm{~s}$ & $35.6,59.6,63.0$ \\
$\mathrm{CH}_{3}$ & 20.5 & $1.77 \mathrm{dq}$ & $126.7,140.3,166.1$ \\
$\mathrm{CH}_{3}$ & 20.9 & $1.98 \mathrm{~s}$ & 170.4 \\
$\mathrm{CH}_{2}$ & 24.8 & $2.95 \mathrm{~m}, 2.43 \mathrm{~m}$ & $35.6,59.6($ débil), $130.2,149.5$, \\
& & & $17.7,24.8,59.6,63.0,76.3$ (débil), 149.5 \\
$\mathrm{CH}_{2}$ & 35.6 & $2.32 \mathrm{~m}, 1.20 \mathrm{~m}$ & $17.7,24.8,59.6,149.5$ \\
$\mathrm{CH}$ & 45.7 & $2.98 \mathrm{~m}$ & $123.1,76.3,59.6,63.0$ \\
$\mathrm{CH}$ & 52.6 & $3.81 \mathrm{~s}$ & 165.7 \\
$\mathrm{C}$ & 59.6 & & \\
$\mathrm{CH}$ & 63.0 & $2.67 \mathrm{~d}$ & $35.6,45.7,59.6,76.3$ \\
$\mathrm{CH}$ & 69.5 & $6.71 \mathrm{dd}$ & $45.7,70.8,76.3,133.5,166.1$ \\
$\mathrm{C}$ & 70.8 & $5.84 \mathrm{~d}$ & $69.5,130.2,149.5,165.7,170.4$ \\
$\mathrm{CH}$ & 76.3 & $4.26 \mathrm{t}$ & $45.7,59.6,63.0,69.5$ \\
$\mathrm{CH}$ & 123.1 & $5.90 \mathrm{~d}, 6.32 \mathrm{~d}$ & $45.7,133.5,168.5$ \\
$\mathrm{C}$ & 126.7 & & \\
$\mathrm{C}$ & 130.2 & & \\
$\mathrm{C}$ & 133.5 & & \\
$\mathrm{CH}$ & 140.3 & $6.08 \mathrm{qq}$ & $24.8,70.8,130.2,165.7$ \\
$\mathrm{CH}$ & 149.5 & $7.14 \mathrm{dd}$ & \\
$\mathrm{C}$ & 165.7 & & \\
$\mathrm{C}$ & 166.1 & & \\
$\mathrm{C}$ & 168.5 & & \\
$\mathrm{C}$ & 170.4 & & \\
\hline
\end{tabular}


Tabla 4. Porcentaje de viabilidad de las células mononucleares de sangre periférica proveniente de donantes con relación al control (DMSO 0.2\%)

\begin{tabular}{|c|c|c|c|c|c|}
\hline \multirow[t]{2}{*}{ Donantes } & \multicolumn{5}{|c|}{ Porcentaje de Viabilidad } \\
\hline & $50 \mu \mathrm{g} / \mathrm{ml}$ & $25 \mu \mathrm{g} / \mathrm{ml}$ & $12.5 \mu \mathrm{g} / \mathrm{ml}$ & $6.0 \mu \mathrm{g} / \mathrm{ml}$ & $3.0 \mu \mathrm{g} / \mathrm{ml}$ \\
\hline 1 & 46 & 70 & 81 & 91 & 108 \\
\hline 2 & 51 & 64 & 72 & 87 & 96 \\
\hline 3 & 53 & 72 & 83 & 95 & 102 \\
\hline 4 & 46 & 62 & 76 & 90 & 98 \\
\hline 5 & 55 & 69 & 85 & 93 & 109 \\
\hline 6 & 58 & 74 & 89 & 97 & 106 \\
\hline 7 & 54 & 77 & 84 & 99 & 109 \\
\hline
\end{tabular}

Tabla 5. Porcentaje de viabilidad en cultivos de células epiteliales sanas con relación al control (DMSO 0.2\%)

\begin{tabular}{cccccc} 
Cultivo Celular & \multicolumn{5}{c}{ Porcentaje de Viabilidad } \\
\hline & $3.0 \mu \mathrm{g} / \mathrm{ml}$ & $6.0 \mu \mathrm{g} / \mathrm{ml}$ & $12.5 \mu \mathrm{g} / \mathrm{ml}$ & $25 \mu \mathrm{g} / \mathrm{ml}$ & $50 \mu \mathrm{g} / \mathrm{ml}$ \\
Tiroides & 85 & 78 & 71 & 69 & 63 \\
Testiculo & 109 & 89 & 73 & 32 & 23 \\
Boca & 94 & 79 & 58 & 35 & 24 \\
\hline
\end{tabular}

Tabla 6. Porcentaje de viabilidad de células mononucleares de sangre periférica proveniente de pacientes con leucemia mieloide crónica y linfoma de Hodking del INC E.S.E de Bogotá - Colombia, con relación al control (DMSO 0.2\%)

\begin{tabular}{cccccc}
\hline Cultivo Celular & \multicolumn{5}{c}{ Porcentaje de Viabilidad } \\
\hline & $3.0 \mu \mathrm{g} / \mathrm{ml}$ & $6.0 \mu \mathrm{g} / \mathrm{ml}$ & $12.5 \mu \mathrm{g} / \mathrm{ml}$ & $25 \mu \mathrm{g} / \mathrm{ml}$ & $50 \mu \mathrm{g} / \mathrm{ml}$ \\
LMC 1 & 43 & 33 & 32 & 30 & 23 \\
LMC 2 & 52 & 38 & 36 & 34 & 29 \\
LH 1 & 56 & 48 & 35 & 28 & 20 \\
LH 2 & 45 & 35 & 32 & 31 & 28 \\
\hline
\end{tabular}

LMC: leucemia mieloide crónica

LH : linfoma de Hodking

cuaternarios; para un total de 23 átomos de carbono en la estructura (Tabla 2). El espectro de masas presenta el ion molecular en 448 , lo cual permite establecer la fórmula $\mathrm{C}_{23} \mathrm{H}_{28} \mathrm{O}_{9}$ para el compuesto.

La elucidación estructural del compuesto se hizo con base en los análisis de los espectros de RMN bidimensionales HMQC y HMBC (Tabla 3). Las constantes de acomplamiento del protón olefínico $\mathrm{H}-1$ en $\delta 7.1(\mathrm{dd}, \mathrm{J}=9.9 ; 9.0 \mathrm{~Hz}$ ), confirman la configuración cis del anillo ciclodecano del melampolido.

Con base en las constantes físicas, los datos espectroscópicos y por comparación con los datos reportados en la literatura, el compuesto se identificó como un germacranólido del tipo melampólido denominado acetato de longipilina (Figura 1).

El compuesto del tipo melampólido: acetato de longipilina fue previamente reportado por Seaman and Fischer en 1978, como el derivado acetilado del compuesto longipilina aislado de la especie Melampodium longipilum, y por Bohlmann et al., 1980 de Smallanthus fruticosus.
Los resultados obtenidos a partir de cultivos de células mononucleares provenientes de donantes sanos se muestran en la tabla 4, Se consideran citotóxicamente activo los porcentajes de viabilidad inferiores a un $50 \%$; lo que demuestra que no presentó un efecto citotóxico importante a concentraciones $\leq$ de $12.5 \mu \mathrm{g} / \mathrm{mL}$, por presentar porcentajes de viabilidad superiores al $80 \%$.

El principio activo acetato de longipilina frente a los cultivos de células epiteliales de boca, testículo y tiroides no presentó un efecto citotóxico relevante a concentraciones $\leq$ a $12.5 \mu \mathrm{g} / \mathrm{mL}$ con porcentajes de viabilidad superiores al $70 \%$ para tiroides y testículo y a concentraciones $\leq$ a $6.0 \mu \mathrm{g} / \mathrm{mL}$ con porcentajes de viabilidad superiores al $78 \%$, como se aprecia en la Tabla 5.

Las células obtenidas de pacientes con leucemia mieloide crónica y linfoma de Hodking fueron sensibles al principio activo, dando como resultado un efecto importante al presentar porcentajes de viabilidad inferiores a un 50\%; a la concentración de $3.0 \mu \mathrm{g} / \mathrm{mL}$, Tabla 6. 


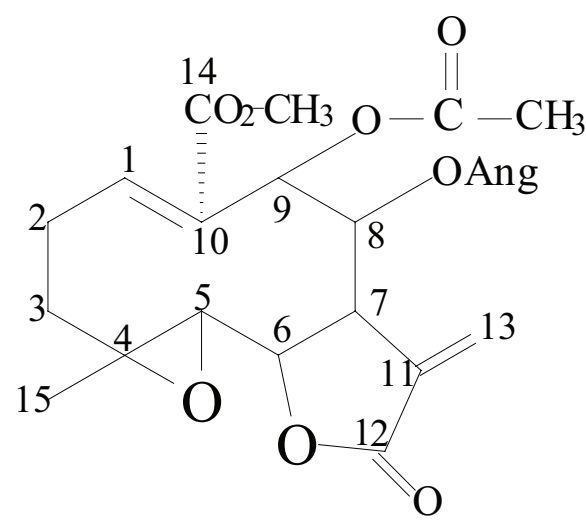

Figura 1. Principio activo citotóxico.

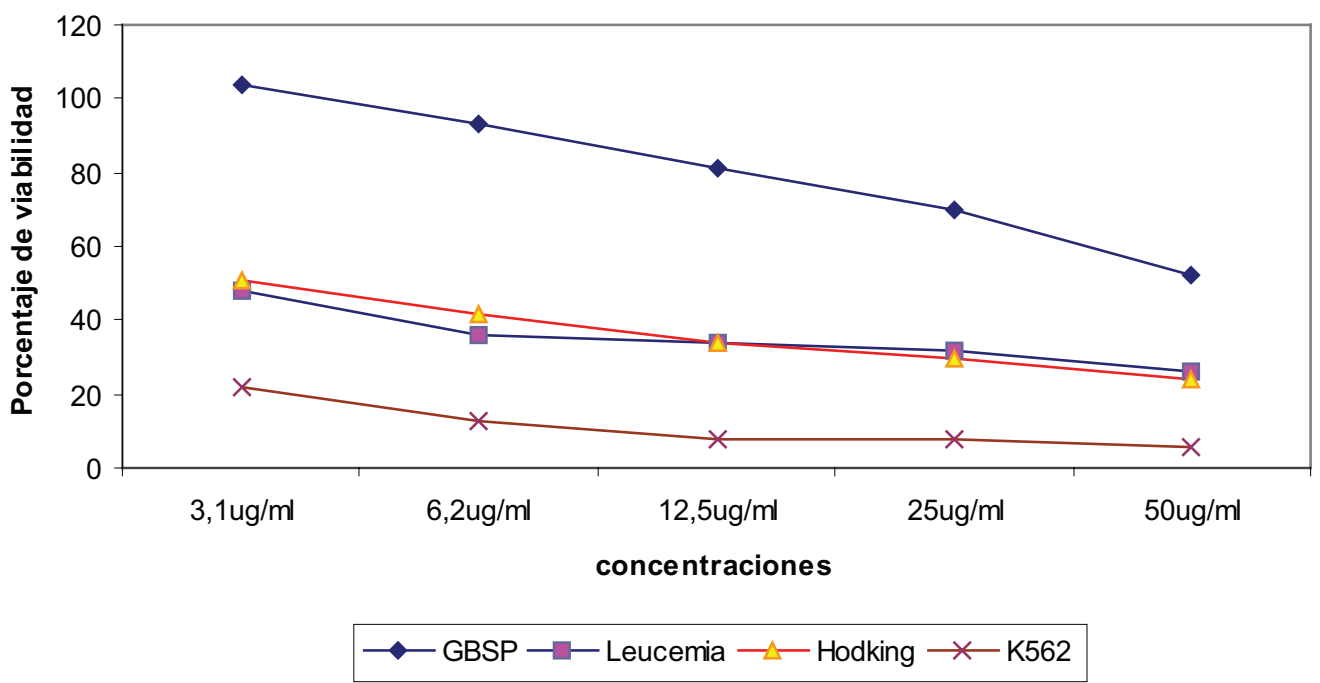

Figura 2. Efecto citotóxico del principio activo. Cada punto representa el promedio de los ensayos en: cultivos de sangre periférica sanos (GBSP), leucemia mieloide crónica, Linfoma de Hodking y la línea celular K562.

El compuesto acetato de longipilina afecta principalmente a los grupos celulares indiferenciados y aumenta cuando su potencial mitótico es mayor como se muestra en la figura 2. Esto concuerda con estudios publicados con sesquiterpenlactonas similares como acetato de ivalina con actividad antileucémica importante y baja toxicidad sobre células mononucleares de sangre periférica (Quintero et al., 1999).

Los resultados de la valoración del efecto del acetato de longipilina sobre cultivos sanos de tiroides, boca y testículo (Figura 3), reveló que la molécula es poco tóxica a concentraciones por debajo de $12.5 \mu \mathrm{g} / \mathrm{mL}$. Estos resultados son importantes teniendo en cuenta que a estas concentraciones el efecto sobre células de pacientes con cáncer y líneas celulares tumorales es tóxica.

En todos los casos se observó significancia estadística (nivel de 0.05). La visión en perspectiva de este trabajo permite proponer que probablemente los cultivos menos diferenciados y más activos en términos de división celular son los más afectados, lo que haría suponer que el principio activo tiene blanco en alguna(s) molécula indispensable para la supervivencia de la célula tumoral. Otras sesquiterpenlactonas similares han demostrado su actividad sobre vías relacionadas con ciclo celular, síntesis del ADN, alteración del metabolismo celular y en la actividad del factor nuclear $N F-\kappa B$ inhibiendo su actividad transcripcional llevando la célula a apoptosis (Lee et al. 1997 y Dirsch et al. 2001).

Finalmente, los resultados demostraron que es una molécula promisoria que amerita ensayos bioquímicos tendientes a revelar los mecanismos moleculares de acción y pruebas sobre modelos animales que evidencien su utilidad como potencial fármaco antitumoral. 


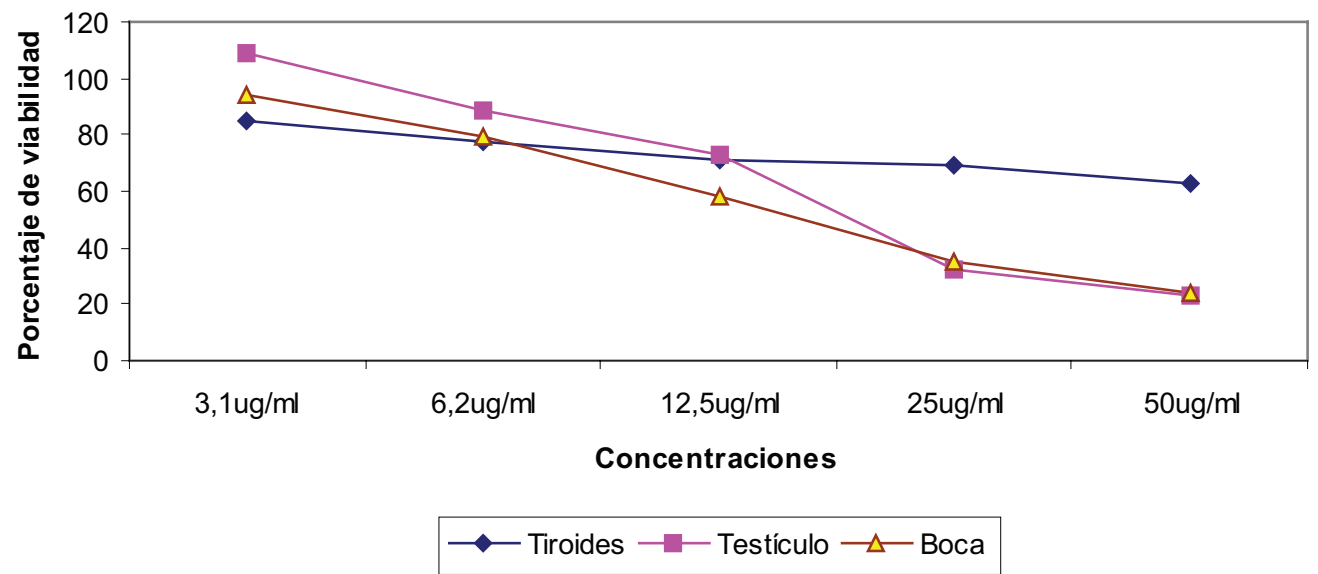

Figura 3. Citotóxicidad del principio activo en cultivos de células de tiroides, testículo y boca, cada punto representa el promedio de los ensayos.

\section{AGRADECIMIENTOS}

Los autores agradecen a COLCIENCIAS, La Pontificia Universidad Javeriana y el Instituto Nacional de Cancerología por el apoyo financiero. Proyecto "Identificación y efecto de posibles sustancias citotóxicas aisladas de especies vegetales colombianas": Col 120305-11460.

\section{REFERENCIAS}

Alfonso ANT, Castro C, Tulia Murcia TR, Torrenegra R 2006. Efectos citotoxicos in vitro de extractos y fracciones de Espeletia killipii Cuatr. frente a lineas celulares tumorales humanos. Rev Bras Farmacogn 16: 12-16.

Bohlmann F, Ziesche J, King R, Robinson H 1980. Neue melampolide aus Smallanthus fruticosus. Phytochemistry 19: 973-974.

Dirsch V, Stuppner H, Vollmar A 2001. Helenanin triggers a CD95 death receptor-independent apoptosis that is not affected by overexpression of Bcl-xl or Bcl-2. Cancer Res 61: 5817-5823.

Huang P, Oliff A 2001. Signaling pathways in apoptosis as potential targets for cancer therapy. Trends Cell Biol 11: 343-348.

Lee K, Hall I, Mar E, Starnes C, Elgebaly S, Waddell T, Hadgraft R, Weidner I 1997. Sesquiterpene antitumor agents: inhibitors of cellular metabolism. Science 196 (4289): 533-536.

Quintero A, Pelcastre A, Dolores J 1999. Antitumoral activity of a new pyrimidine derivatives of sesquiterpene lactones. J Pharmal Sci 2: 108-112.

Seaman F, Fischer N 1978. Longipin, a new melampolide from Melampodium longipilum. Phytochemistry 17: 21312132. 